\title{
Non Self-conjugate Strings, Singular Strings and Rigged Configurations in the Heisenberg Model
}

\author{
Tetsuo Deguch ${ }^{*}$ and Pulak Ranjan Gir \\ Department of Physics, Graduate School of Humanities and Sciences, \\ Ochanomizu University, Ohtsuka 2-1-1, Bunkyo-ku, Tokyo, 112-8610, Japan
}

(Dated: March 27, 2022)

\begin{abstract}
We observe a different type of complex solutions in the isotropic spin- $1 / 2$ Heisenberg chain starting from $N=12$, where the central rapidity of some of the odd-length strings becomes complex making not all the strings self-conjugate individually. We show that there are at most $(N-2) / 2$ singular solutions for $M=4, M=5$ down-spins and at most $\left(N^{2}-6 N+8\right) / 8$ singular solutions for $M=6$, $M=7$ down-spins in an even-length chain with $N \geq 2 M$. Correspondence of the non self-conjugate string solutions and the singular string solutions to the rigged configurations has also been shown.
\end{abstract}

PACS numbers: 71.10.Jm, 02.30Ik, 03.65Fd

\section{INTRODUCTION}

Bethe's solution of the isotropic spin-1/2 Heisenberg model in one dimension, by a method known as the coordinate Bethe ansatz [1, is one of the seminal works in the field of integrable models. For a detailed investigation on the method and its variants and other related works see references [2 11. However, deriving the Bethe ansatz equations and the form of the eigenvalues and eigenvectors is only one part of the story. The other important part is to extract the numerical values of the rapidities from the set of the Bethe ansatz equations. Because of the high degree of nonlinearity and multi-variate nature it is practically impossible to analytically solve the Bethe ansatz equations even for a modest length of the spin chain. One, therefore needs to seek numerical solutions using methods, such as, iterations, the Newton-Raphson, homotopy continuations etc. There have been some efforts to find the numerical solutions of the Bethe ansatz equations using different techniques, and the eigenvalues have been found which have excellent match with direct diagonalization of the Hamiltonian.

Apart from the real solutions, which are much easier to find out, there are complex solutions of the Bethe ansatz equations, which need extra efforts to calculate. Bethe himself investigated this problem for a finite length spin chain and found that if there are complex solutions then they come in complex conjugate pairs and arrange themselves in a string-like structure [1]. These complex solutions, responsible for the formation of bound states, lead to the so-called string hypothesis [12]. Importance of numerical computations of all the Bethe solutions are in one hand to check the completeness of the spectrum of the Hamiltonian and on the other hand the knowledge of the numerical solutions are necessary for the computation of correlation functions, form factors [13, 14] and other physical quantities of the model.

Although, the string hypothesis gives satisfactory results in the thermodynamic limit and counts the total number of states correctly in the general case, it has many drawbacks and there have been found some exceptions to it. For example, if the string hypothesis is valid in all respects then for a large length chain the imaginary part of the rapidities of 2-string should converges to $\pm 1 / 2$. However it has been shown in [15] that some of the 2-string rapidities $\lambda_{ \pm}$, behave as $\operatorname{Re}\left(\lambda_{ \pm}\right) \sim N, \operatorname{Im}\left(\lambda_{ \pm}\right) \sim \pm \sqrt{N}$ for large length $N$ spin chains. Even, there are some 2-strings which for large $N$ and large Bethe quantum numbers deform back to two real rapidities [16-19], which is observed numerically. Despite these drawbacks, the string hypothesis has been very helpful in numerical analysis in the iteration method to obtain good initial guess for the finite length chains. Exploiting the string hypothesis and taking into account the deformations, the complete string solutions for $N=8$ and $N=10$ length isotropic spin- $1 / 2$ Heisenberg chains are obtained in [20]. String solutions up to $N=14$ have been obtained in 21] and its supplement by making use of the homotopy continuation method to show that there are too many solutions of the Bethe ansatz equations and only some of them, which obey the self-conjugacy condition, are the physical solutions of the Heisenberg model. Here we remark that we call a solution of the Bethe ansatz equations physical if it leads to an eigenvector of the Hamiltonian.

Usually, a set of solutions to the Bethe ansatz equations consists of strings of different lengths. As mentioned above, one key constraint to the solutions of the Bethe ansatz equations is that they are self-conjugate [22]. While

*Electronic address: deguchi@phys.ocha.ac.jp

${ }^{\dagger}$ Electronic address: pulakgiri@gmail.com 
implementing this constraint in the string hypothesis it is usually assumed that self-conjugacy is to be satisfied within a string [20], a condition, motivated by the observation of the short length spin chains, is too restrictive to be valid for all solutions of higher-length spin chains. We therefore relax the imposition of the self-conjugacy criterion to the whole set of rapidities not necessarily within a string, making the strings in a solution individually not self-conjugate. We show that our self-conjugacy criterion allows us to obtain some solutions which are not fitted within the standard deformed string picture, which is one of our motivations in this work. For even-length chains, up to $N=10$ the string solutions, although deformed, still do obey the string structure and the restrictive self-conjugacy condition. First breakdown of the string structure for the physical solutions of even-length chains occurs in $N=12$, as some of the strings become non self-conjugate and therefore need the most relaxed self-conjugacy condition. We discuss this feature of the string solutions here with an example of $N=12$. Although numerical solutions for $N=12$ is obtained in [21] using the homotopy continuation method we here obtained the solutions by the iteration method using Mathematica and exploiting the string hypothesis. Here we remark that some different types of solutions of the Bethe ansatz equations in the anisotropic Heisenberg models are studied in [23 26].

Moreover, recently a lot of works on the physical singular solutions have been reported in the literature [21, 27 29]. The singular solutions as we know are an essential component of the spectrum and need a proper regularization scheme to derive the correct physical states and the eigenvalues. It is also possible to map these solutions and even the regular solutions to a type of combinatorial objects known as rigged configurations [30 32]. Based on the symmetry of the singular solutions for even-length chains we classify the solutions in different categories, which allow us to simplify the Bethe ansatz equations significantly up to $M=7$ down-spins. Studying the algebraic aspects of polynomials we estimate the number of singular solutions present at most for even-length spin chains up to $M=7$ down-spins. We also study the aspect of mapping of the Bethe states to rigged configurations for singular solutions as well as solutions with non self-conjugate strings.

Our study enables us to identify all the physical singular solutions present in the $N=12$ case and map them to rigged configurations. It also simplifies the Bethe ansatz equations for the singular solutions significantly up to $M=7$ down-spins with even $N$ so that the physical singular solutions and their total number are obtained systematically.

We organize this paper in the following fashion: In the next section, we briefly present the isotropic spin- $1 / 2$ chain and its solutions in terms of the algebraic Bethe ansatz method. In section III, we discuss the non self-conjugate strings and explain them with the example of $N=12$. In section IV, we discuss the singular solutions, their classification and give an estimate of the number singular solutions. In section $\mathrm{V}$, we discuss rigged configurations and their correspondence with $N=12$ case for non self-conjugate string solutions and singular string solutions and finally we conclude.

\section{ALGEBRAIC BETHE ANSATZ}

The Hamiltonian of a spin- $1 / 2$ chain with length $N$ under the periodic boundary conditions is given by

$$
H=J \sum_{i=1}^{N}\left(S_{i}^{x} S_{i+1}^{x}+S_{i}^{y} S_{i+1}^{y}+S_{i}^{z} S_{i+1}^{z}-1 / 4\right),
$$

where $J$ is the coupling constant and $S_{i}^{j}(j=x, y, z)$ the spin at position $i$ and in $j$-direction. In the algebraic Bethe ansatz formulation one can construct a Bethe state in the case of $M$ down spin sector as

$$
\left|\lambda_{1}, \lambda_{2}, \cdots, \lambda_{M}\right\rangle=\prod_{\alpha=1}^{M} B\left(\lambda_{\alpha}\right)|\Omega\rangle,
$$

from the reference state $|\Omega\rangle$ with all up spins by acting the $B\left(\lambda_{\alpha}\right)$ matrix. To obtain the $B\left(\lambda_{\alpha}\right)$ matrix we need the Lax operator

$$
L_{\gamma}(\lambda)=\left(\begin{array}{cc}
\lambda-i S_{\gamma}^{z} & -i S_{\gamma}^{-} \\
-i S_{\gamma}^{+} & \lambda+i S_{\gamma}^{z}
\end{array}\right)
$$

where $S_{\gamma}^{ \pm}=S_{\gamma}^{x} \pm i S_{\gamma}^{y}$ are the Pauli spin-1/2 matrixes. Each element of this matrix acts nontrivially on the $\gamma$-th lattice site of the Heisenberg model. One can get $B\left(\lambda_{\alpha}\right)$ from the monodromy matrix

$$
T(\lambda)=L_{N}(\lambda) L_{N-1}(\lambda) \cdots L_{1}(\lambda)=\left(\begin{array}{cc}
A(\lambda) & B(\lambda) \\
C(\lambda) & D(\lambda)
\end{array}\right)
$$


The Bethe state 20 can also be written in a very useful form as 33

$$
\begin{aligned}
\prod_{\alpha=1}^{M} B\left(\lambda_{\alpha}\right)|\Omega\rangle= & (-2 i)^{M} \prod_{j<k}^{M} \frac{\lambda_{j}-\lambda_{k}+i}{\lambda_{j}-\lambda_{k}} \prod_{j=1}^{M} \frac{\left(\lambda_{j}-i / 2\right)^{N}}{\lambda_{j}+i / 2} \times \\
& \sum_{1 \leq x_{1}<x_{2} \ldots \ldots<x_{M} \leq N}^{N} \sum_{\mathcal{P} \in S_{M}}^{M !} \prod_{\mathcal{P} j<\mathcal{P} k}^{M}\left(\frac{\lambda_{\mathcal{P} j}-\lambda_{\mathcal{P} k}-i}{\lambda_{\mathcal{P} j}-\lambda_{\mathcal{P} k}+i}\right)^{H(j-k)} \prod_{j=1}^{M}\left(\frac{\lambda_{\mathcal{P} j}+i / 2}{\lambda_{\mathcal{P} j}-i / 2}\right)^{x_{j}} \prod_{j=1}^{M} S_{x_{j}}^{-}|\Omega\rangle,
\end{aligned}
$$

where $\mathcal{P}$ are elements of the permutation group $S_{M}$ of the $M$ numbers and $H(x)$ is the Heaviside step function $H(x)=1$ for $x>0$ and $H(x)=0$ for $x \leq 0$.

When the rapidities $\lambda_{\alpha}$ satisfy the well known Bethe ansatz equations

$$
\left(\frac{\lambda_{\alpha}-i / 2}{\lambda_{\alpha}+i / 2}\right)^{N}=\prod_{\beta \neq \alpha}^{M} \frac{\lambda_{\alpha}-\lambda_{\beta}-i}{\lambda_{\alpha}-\lambda_{\beta}+i} \quad \alpha=1,2, \cdots, M,
$$

then the Bethe state given by eq. (2) ( and (5)) becomes the highest-weight Bethe eigenstate. We also call the solutions of (6) the Bethe roots. The eigenvalue of the Hamiltonian (1) for the $M$ down-spin configuration is then given by

$$
E=-J \frac{1}{2} \sum_{\alpha=1}^{M} \frac{1}{\left(\lambda_{\alpha}^{2}+1 / 4\right)}
$$

A convenient way to deal with the Bethe ansatz equations is to take the logarithm of eq. (6)

$$
2 \arctan \left(2 \lambda_{\alpha}\right)=J_{\alpha} \frac{2 \pi}{N}+\frac{2}{N} \sum_{\beta \neq \alpha}^{M} \arctan \left(\lambda_{\alpha}-\lambda_{\beta}\right) \quad \bmod 2 \pi,
$$

where the Bethe quantum numbers, $\left\{J_{\alpha}, \alpha=1,2, \cdots, M\right\}$, take integral or half integral values, depending on whether $N-M$ is odd or even, respectively. However, since $J_{\alpha}$ are repetitive in a given state, it is not useful for counting the number of states of the model in concern. But, it is possible to obtain non-repetitive quantum numbers with the help of the string hypothesis, which says that the rapidities for $M$ down-spins are arranged in a set of strings as

$$
\lambda_{\alpha a}^{j}=\lambda_{\alpha}^{j}+\frac{i}{2}(j+1-2 a)+\Delta_{\alpha a}^{j} \quad a=1,2, \cdots, j, \quad \alpha=1,2, \cdots,
$$

where the string center $\lambda_{\alpha}^{j}$ is real, $j$ is the length of the string, $\alpha$ accounts for the number of the $j$-strings and $\Delta_{\alpha a}^{j}$ are the string deviations. In the limit $\Delta_{\alpha a}^{j} \rightarrow 0$, the Bethe ansatz equations [6] reduce to [12]

$$
\begin{aligned}
\arctan \frac{2 \lambda_{\alpha}^{j}}{j} & =\pi \frac{I_{\alpha}^{j}}{N}+\frac{1}{N} \sum_{k=1}^{N_{s}} \sum_{\beta}^{M_{k}} \Theta_{j k}\left(\lambda_{\alpha}^{j}-\lambda_{\beta}^{k}\right) \quad \bmod \pi \\
\Theta_{j k}(\lambda) & =\left(1-\delta_{j k}\right) \arctan \frac{2 \lambda}{|j-k|}+2 \arctan \frac{2 \lambda}{|j-k|+2}+\cdots+2 \arctan \frac{2 \lambda}{j+k-2}+\arctan \frac{2 \lambda}{j+k},
\end{aligned}
$$

where $M_{k}$ is the number of $k$-strings present in the $M$ down-spin state and $N_{s}$ is the length of the largest string such that $\sum_{k}^{N_{s}} k M_{k}=M$. The Takahashi quantum numbers $I_{\alpha}^{j}$, which are non-repetitive, are given by

$$
\left|I_{\alpha}^{j}\right| \leq \frac{1}{2}\left(N-1-\sum_{k=1}\left[2 \min (j, k)-\delta_{j, k}\right] M_{k}\right)
$$

\section{NON SELF-CONJUGATE STRINGS}

In this section we present the non-string type solutions, which start to occur from the $N=12$ case. One of the important ingredients for an effective iteration method is to start the numerical procedure with a very good initial 
TABLE I: Non self-conjugate Bethe roots for $N=12, M=5$. There are only 2 solutions with two 1 -strings and one 3-string

\begin{tabular}{|c|c|c|c|c|c|c|c|}
\hline & $\overline{J J}$ & 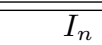 & $\overline{\lambda \lambda}$ & $\bar{E}$ & \multicolumn{3}{|c|}{ Rigged Configuration } \\
\hline \multirow{5}{*}{1.} & 2 & $3 / 2_{1}$ & 0.18071431863183055 & \multirow{5}{*}{-3.6006932562693255} & & & \multirow{5}{*}{2} \\
\hline & 3 & \multirow{2}{*}{$5 / 2_{1}$} & $0.44476350644863927-0.01877019940237738 \mathrm{i}$ & & 2 & & \\
\hline & 4 & & $0.49181421369589934+0.9614711323790809 \mathrm{i}$ & & 6 & 5 & \\
\hline & 3 & \multirow[t]{2}{*}{$1_{3}$} & $0.44476350644863927+0.01877019940237738 \mathrm{i}$ & & 6 & \multirow{2}{*}{5} & \\
\hline & 5 & & $0.49181421369589934-0.9614711323790809 \mathrm{i}$ & & & & \\
\hline \multirow{5}{*}{2.} & -2 & $-3 / 2_{1}$ & -0.18071431863183055 & \multirow{5}{*}{-3.6006932562693255} & & & \multirow{5}{*}{0} \\
\hline & -3 & $-5 / 2_{1}$ & $-0.44476350644863927+0.01877019940237738 \mathrm{i}$ & & 2 & & \\
\hline & -4 & \multirow{3}{*}{$-1_{3}$} & $-0.49181421369589934-0.9614711323790809 \mathrm{i}$ & & 6 & \multirow{3}{*}{$\begin{array}{l}1 \\
1\end{array}$} & \\
\hline & -3 & & $-0.44476350644863927-0.01877019940237738 \mathrm{i}$ & & 6 & & \\
\hline & -5 & & $-0.49181421369589934+0.9614711323790809 \mathrm{i}$ & & & & \\
\hline
\end{tabular}

TABLE II: Non self-conjugate Bethe roots for $N=12, M=6$. There are three solutions with one 1-string, one 2-string and one 3 -string

\begin{tabular}{|c|c|c|c|c|c|c|c|c|}
\hline & $J$ & $I_{n}$ & $\lambda$ & $E$ & Rigg & $\overline{\mathrm{Con}}$ & $\overline{\text { figl }}$ & ration \\
\hline & $-1 / 2$ & $0_{1}$ & $0.0185398999059037 i$ & & & & & \\
\hline & $5 / 2$ & $0_{2}$ & $0.5 \mathrm{i}$ & & 0 & & & 0 \\
\hline 1. & $7 / 2$ & & $-0.5 \mathrm{i}$ & -3.6497381892472 & 2 & & 1 & \\
\hline & $1 / 2$ & $0_{3}$ & $-0.0185398999059037 \mathrm{i}$ & & 6 & 3 & & \\
\hline & $-9 / 2$ & & $-0.993775005875478 \mathrm{i}$ & & & & & \\
\hline & $3 / 2$ & $2_{1}$ & $0.384905215843542+0.0190612670356019 \mathrm{i}$ & & & & & \\
\hline & $-7 / 2$ & -1 , & $-0.752213256639834+0.507293831282871 \mathrm{i}$ & & 0 & & & 0 \\
\hline 2. & $\begin{array}{r}-5 / 2 \\
0 / 2\end{array}$ & $-I_{2}$ & $-0.752213256639834-0.507293831282871 \mathrm{i}$ & -2.46168458170981 & 2 & & 0 & \\
\hline & $3 / 2$ & $0_{3}$ & $0.384905215843542-0.0190612670356019 \mathrm{i}$ & & 6 & 5 & & \\
\hline & $11 / 2$ & & $0.367308040796292-0.991797190897116 \mathrm{i}$ & & & & & \\
\hline & $-3 / 2$ & $-2_{1}$ & $-0.384905215843542+0.0190612670356019 \mathrm{i}$ & & & & & \\
\hline & $7 / 2$ & 1 & $0.752213256639834-0.507293831282871 \mathrm{i}$ & & 0 & & & 0 \\
\hline 3. & $\begin{array}{r}5 / 2 \\
-9 / 2\end{array}$ & $1_{2}$ & $\begin{array}{l}0.752213256639834+0.507293831282871 \mathrm{i} \\
-0367308040796292-0.991797190897116 \mathrm{i}\end{array}$ & -2.46168458170981 & 2 & & 2 & \\
\hline & $-3 / 2$ & $0_{3}$ & $-0.384905215843542-0.0190612670356019 \mathrm{i}$ & & 6 & 1 & & \\
\hline & $-11 / 2$ & & $-0.367308040796292+0.991797190897116 \mathrm{i}$ & & & & & \\
\hline
\end{tabular}

guess. In case of the Bethe equations (6) it can be found by solving the Takahashi equations (10). One then needs to find the deviations $\Delta_{\alpha a}^{j}$ of the string to obtain the actual roots. In the string hypothesis these deviations are supposed to be purely imaginary and decrease exponentially with respect to $N$. In finite-size spin chains, however, there are deviations of the string center, which leads to the form 20]

$$
\Delta_{\alpha a}^{j}=\epsilon_{\alpha a}^{j}+i \delta_{\alpha a}^{j}
$$

where $\epsilon_{\alpha a}^{j}$ and $\delta_{\alpha a}^{j}$ are real. Since the Bethe roots are self-conjugate there should be a corresponding restriction on the deviations. One choice is to consider self-conjugacy within a string of length $j$, which translates to $\Delta_{\alpha a}^{j}=\left(\Delta_{\alpha j+1-a}^{j}\right)^{*}$. A consequence of this picture is that the central rapidity of an odd-length string is always real and there is as such no relation between different strings in an eigenstate. For $N=8$ and $N=10$ we can recover all the string solutions from this consideration of string picture and it is therefore sufficient to restrict the self-conjugacy condition within a string. However, as we increase the length $N$ of the spin chain it is not possible to recover all the string solutions since the above self-conjugacy condition is too restrictive if we assume that deviations are small. Note that in the string hypothesis it is crucial to construct the strings in such a way that the deviations 12 are small enough so that the $\Delta_{\alpha a}^{j} \rightarrow 0$ limit leads to an approximate solution of the Bethe ansatz equations, which satisfies the Takahashi equations (10) and gives the Takahashi quantum numbers (11). One therefore needs to impose the self-conjugacy condition on the whole set of strings $\left\{\lambda_{\alpha a}^{j}\right\}=\left\{\left(\lambda_{\alpha a}^{j}\right)^{*}\right\}$ as mentioned in introduction. Keeping the deviations small 


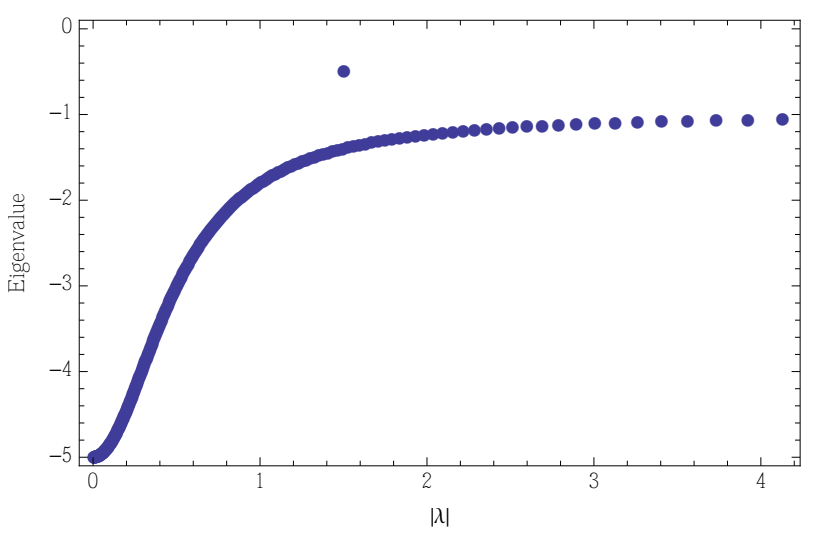

FIG. 1: The energy eigenvalues for the singular solutions for $N=500, M=4$ with absolute value $|\lambda|$ of the form, $a_{1}$ or $i a_{1}$ of eqs. 22 and 23 , respectively. Up to $N=500$, we obtained only one solution of the form $i a_{1}$ for every even-integer $N \geq 8$, with $i a_{1} \rightarrow 1.5 i$ for large $N$ and the corresponding eigenvalue $E \rightarrow-0.5$ as can be seen from the isolated point in the figure

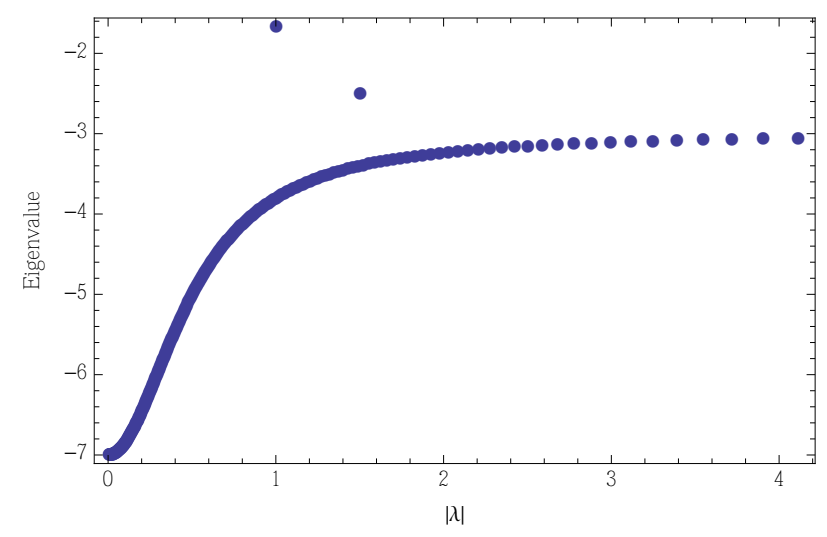

FIG. 2: The energy eigenvalues for the singular solutions for $N=500, M=5$ with absolute value $|\lambda|$ of the form, $a_{1}$ or $i a_{1}$ of eqs. 30 and 31 , respectively. Up to $N=500$ we obtained only two solutions of the form $i a_{1}$ for every even $N \geq 10$, with $i a_{1} \rightarrow 1.5 i$ and $i a_{1} \rightarrow 1 i$, respectively for large $N$ and the corresponding eigenvalues, $E \rightarrow-2.5$ and $E \rightarrow-1.6666666666666667$, respectively as can be seen from the two isolated points in the figure

and relaxing the self-conjugacy to the whole set of strings, we obtain some solutions, which are not self-conjugate, we call such solutions non self-conjugate string solutions.

To clarify the point with concrete examples let us consider a state of $M=5$ down-spins in a $N=12$ spin chain, with two 1-strings $\left(M_{1}=2\right)$ and one 3 -string $\left(M_{3}=1\right)$. If we consider the self-conjugacy condition only within each string then the Bethe roots can be parametrized as

$$
\left\{\lambda_{1}\right\},\left\{\lambda_{2}\right\},\{\lambda+\epsilon+(1+\delta) i, \lambda, \lambda+\epsilon-(1+\delta) i\},
$$

where $\lambda_{1}, \lambda_{2}, \lambda, \epsilon, \delta$ are real parameters which we have to evaluate numerically. We put curly brackets to separate different strings. All the physical solutions for $N=12, M=5, M_{1}=2, M_{3}=1$ though fall in this category except two solutions in which case the roots can be parametrized as

$$
\left\{\lambda_{1}\right\},\left\{\lambda+i \delta_{1}\right\},\left\{\lambda+\epsilon+\left(1+\delta_{2}\right) i, \lambda-i \delta_{1}, \lambda+\epsilon-\left(1+\delta_{2}\right) i\right\},
$$

where $\lambda_{1}, \lambda, \epsilon, \delta_{1}, \delta_{2}$ are real parameters. In Table I two such solutions of this kind are shown where one of the two 1-strings becomes complex conjugate to the central rapidity of the three string and therefore the 1-string and the 3 -string become non self-conjugate individually but remains self-conjugate when considered collectively. In all the tables $I_{n}$ represent the Takahashi quantum numbers for the $n$-strings.

Another example is present in the $M=6$ down spin sector, with one 1-string $\left(M_{1}=1\right)$, one 2 -string $\left(M_{2}=1\right)$ and one 3-string $\left(M_{3}=1\right)$. Again if we consider self-conjugacy for each string then the Bethe roots can be parametrized 
TABLE III: Singular Bethe roots for $N=12, M=2$. There is one solution with one 2-string

\begin{tabular}{|c|c|c|c|c|c|c|}
\hline & $J$ & $I_{n}$ & $\lambda$ & $E$ & Rigg & iguration \\
\hline 1. & $\begin{array}{l}5 / 2 \\
7 / 2\end{array}$ & $0_{2}$ & $\begin{array}{l}0.5 \mathrm{i} \\
-0.5 \mathrm{i}\end{array}$ & -1.0 & 8 & 4 \\
\hline
\end{tabular}

TABLE IV: Singular Bethe roots for $N=12, M=3$. There is one solution with one 1-string and one 2-string

\begin{tabular}{|c|c|c|c|c|c|c|}
\hline & & $\overline{\overline{I_{n}}}$ & $\overline{\lambda \lambda}$ & $\overline{E E}$ & \multicolumn{2}{|c|}{ Rigged Configuration } \\
\hline \multirow[b]{2}{*}{1.} & 0 & $0_{1}$ & 0.0 & & 6 & 3 \\
\hline & $\begin{array}{l}3 \\
3\end{array}$ & $0_{2}$ & $\begin{array}{l}0.5 \mathrm{i} \\
-0.5 \mathrm{i}\end{array}$ & -3.0 & 8 & 4 \\
\hline
\end{tabular}

as

$$
\left\{\lambda_{2}\right\},\left\{\lambda_{1}+\frac{i}{2}(1+2 \delta), \lambda_{1}-\frac{i}{2}(1+2 \delta)\right\},\left\{\lambda+\epsilon+\left(1+\delta_{1}\right) i, \lambda, \lambda+\epsilon-\left(1+\delta_{1}\right) i\right\},
$$

where $\lambda_{1}, \lambda_{2}, \lambda, \delta, \delta_{1}, \epsilon$ are real parameters. All the solutions fall in this category except the three solutions which follow

$$
\left\{\lambda+i \delta_{1}\right\},\left\{\lambda_{1}+\frac{i}{2}(1+2 \delta), \lambda_{1}-\frac{i}{2}(1+2 \delta)\right\},\left\{\lambda+\epsilon+\left(1+\delta_{2}\right) i, \lambda-i \delta_{1}, \lambda+\epsilon-\left(1+\delta_{2}\right) i\right\},
$$

where $\lambda, \lambda_{1}, \delta_{2}, \delta, \delta_{1}, \epsilon$ are real parameters. We can still use (14) and (16) to get a relation between the Bethe quantum numbers $J_{i}$ and the Takahashi quantum numbers $I_{n}^{\alpha}$ by first simply taking $\delta_{1} \rightarrow 0$ limit in the expressions of the two rapidities $\lambda+i \delta_{1}$ and $\lambda-i \delta_{1}$. We note that in the $\delta_{1} \rightarrow 0$ limit the two rapidities do not become equal to each other, rather they go to two distinct rapidities, $\lim _{\delta_{1} \rightarrow 0} \lambda+i \delta_{1} \rightarrow \lambda_{2}$ and $\lim _{\delta_{1} \rightarrow 0} \lambda-i \delta_{1} \rightarrow \lambda$. Then (14) and (16) reduces to the standard string roots of (13) and (15) respectively, which allows us to obtain the Takahashi quantum numbers in terms of the Bethe quantum numbers. In Table II the three solutions of the form (16) have been shown.

\section{SINGULAR STRINGS}

Singular string solutions of the Bethe equations are special in the sense that the energy eigenvalue diverges and the Bethe state vanishes without regularization and therefore we need to have a suitable regularization scheme [10, 27-

\begin{tabular}{|c|c|c|c|c|c|c|c|}
\hline & 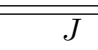 & $\overline{\overline{I_{n}}}$ & $\overline{\bar{\lambda}}$ & $\overline{E E}$ & \multicolumn{3}{|c|}{ Rigged Configuration } \\
\hline \multirow{3}{*}{1.} & $-7 / 2$ & $-7 / 2_{1}$ & -1.1657640741782098 & \multirow{3}{*}{-1.6215017698290826} & 4 & & \multirow[t]{3}{*}{2} \\
\hline & $\begin{array}{l}7 / 2 \\
5 / 2\end{array}$ & $7 / 2_{1}$ & $\begin{array}{l}1.1657640741782098 \\
0.5 \mathrm{i}\end{array}$ & & 6 & 0 & \\
\hline & $7 / 2$ & $0_{2}$ & $-0.5 \mathrm{i}$ & & 6 & 6 & \\
\hline \multirow{3}{*}{2.} & $-5 / 2$ & $-5 / 2_{1}$ & -0.535523144483441 & \multirow{3}{*}{-2.8629431312188682} & 4 & & \multirow[t]{3}{*}{2} \\
\hline & $5 / 2$ & $5 / 2_{1}$ & 0.535523144483441 & & 6 & 1 & \\
\hline & $7 / 2$ & $\mathrm{O}_{2}$ & $-0.5 \mathrm{i}$ & & 6 & 5 & \\
\hline \multirow{3}{*}{3.} & $-3 / 2$ & $-3 / 2_{1}$ & -0.2659137285956084 & \multirow{3}{*}{-4.118080676373112} & 4 & & \multirow[t]{3}{*}{2} \\
\hline & $\begin{array}{l}3 / 2 \\
5 / 2\end{array}$ & $3 / 2_{1}$ & 0.2659137285956084 & & 6 & 2 & \\
\hline & $7 / 2$ & $0_{2}$ & $-0.5 \mathrm{i}$ & & 6 & 4 & \\
\hline \multirow{3}{*}{4.} & $-1 / 2$ & $-1 / 2_{1}$ & -0.08199356634008453 & \multirow{3}{*}{-4.895249800546962} & 4 & & \multirow[t]{3}{*}{2} \\
\hline & $1 / 2$ & $1 / 2_{1}$ & 0.08199356634008453 & & 6 & 3 & \\
\hline & & $0_{2}$ & $\begin{array}{l}0.51 \\
-0.5 \mathrm{i}\end{array}$ & & 6 & 3 & \\
\hline
\end{tabular}

TABLE V: Singular Bethe roots for $N=12, M=4$. There are four solutions with two 1-strings and one 2-string 
TABLE VI: Singular Bethe roots for $N=12, M=4$. There is one solution with one 4-string

\begin{tabular}{|c|c|c|c|c|c|c|}
\hline \multirow{4}{*}{1.} & 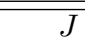 & $\overline{I_{n}}$ & $\overline{\lambda \lambda}$ & $\bar{E}$ & Rigg & \\
\hline & $9 / 2$ & \multirow{3}{*}{$0_{4}$} & $1.502976465754898 \mathrm{i}$ & \multirow{3}{*}{-0.5022246220319766} & \multirow{3}{*}{4} & \multirow{3}{*}{2} \\
\hline & $\begin{array}{l}5 / 2 \\
7 / 2\end{array}$ & & $\begin{array}{l}0.5 \mathrm{i} \\
-0.5 \mathrm{i}\end{array}$ & & & \\
\hline & $-9 / 2$ & & $-1.502976465754898 \mathrm{i}$ & & & \\
\hline
\end{tabular}

TABLE VII: Singular Bethe roots for $N=12, M=5$. There are three solutions with three 1 -strings and one 2 -string

\begin{tabular}{|c|c|c|c|c|c|c|c|}
\hline & $\overline{\bar{J}}$ & 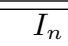 & $\overline{\lambda \lambda}$ & $\bar{E}$ & \multicolumn{3}{|c|}{ "Rigged Configuration } \\
\hline \multirow{5}{*}{1.} & 0 & $0_{1}$ & 0.0 & \multirow{5}{*}{-3.9168598958278036} & 2 & & \multirow[t]{5}{*}{1} \\
\hline & -3 & $-3_{1}$ & -0.9168855875745959 & & & & \\
\hline & 3 & $3_{1}$ & 0.9168855875745959 & & 4 & 0 & \\
\hline & 3 & \multirow{2}{*}{$0_{2}$} & $0.5 \mathrm{i}$ & & 4 & 2 & \\
\hline & 3 & & $-0.5 \mathrm{i}$ & & 4 & 4 & \\
\hline \multirow{5}{*}{2.} & 0 & $0_{1}$ & 0.0 & \multirow{5}{*}{-5.3599633111443925} & 2 & & \multirow[t]{5}{*}{1} \\
\hline & -2 & $-2_{1}$ & -0.4168157878523103 & & & 1 & \\
\hline & 2 & $2{ }_{1}$ & 0.4168157878523103 & & 4 & \multirow{2}{*}{$\begin{array}{l}1 \\
2\end{array}$} & \\
\hline & 3 & \multirow{2}{*}{$0_{2}$} & $0.5 \mathrm{i}$ & & 4 & & \\
\hline & 3 & & $-0.5 \mathrm{i}$ & & 4 & 3 & \\
\hline \multirow{5}{*}{3.} & 0 & $0_{1}$ & 0.0 & \multirow{5}{*}{-6.545681807497121} & 2 & & \multirow[t]{5}{*}{1} \\
\hline & -1 & $-1_{1}$ & -0.1789782217190064 & & & \multirow{2}{*}{2} & \\
\hline & 1 & $1_{1}$ & 0.1789782217190064 & & 4 & & \\
\hline & 3 & \multirow{2}{*}{$0_{2}$} & $0.5 \mathrm{i}$ & & 4 & 2 & \\
\hline & 3 & & $-0.5 \mathrm{i}$ & & 4 & 2 & \\
\hline
\end{tabular}

22, 36 to make everything finite. It is also an essential part of the spectrum because without the singular solutions the Hilbert space of the Hamiltonian is not complete. Recently there has been much interest in singular solutions and it is also possible to map all the singular solutions to rigged configurations. Solutions of the form

$$
\left\{\lambda_{1}=\frac{i}{2}, \lambda_{2}=-\frac{i}{2}, \lambda_{3}, \lambda_{4}, \cdots, \lambda_{M}\right\},
$$

are called singular because two of the roots $\lambda_{1}, \lambda_{2}$ make the state and the corresponding eigenvalue ill-defined. It has been numerically observed that the roots of a singular state for even $N$ are distributed symmetrically, we assume that for the physical singular solutions the following condition is satisfied

$$
\sum_{\alpha=1}^{M} \lambda_{\alpha}=0 .
$$

The condition 18 is satisfied for any symmetrically distributed roots, which may or may not be singular. We remark that the condition (18) has been conjectured in 35, for even length spin chain. Another point is that the singular solutions in [27] for even $N$, which do not satisfy the condition [18), are not physical. In singular 2-string $\left\{ \pm \frac{i}{2}\right\}$ case

\begin{tabular}{|c|c|c|c|c|c|c|c|}
\hline & $\overline{J J}$ & $\overline{I_{n}}$ & $\bar{\lambda}$ & $\bar{E}$ & \multicolumn{3}{|c|}{ Rigged Configuration } \\
\hline \multirow{4}{*}{1.} & 0 & $0_{1}$ & 0.0 & & & & \multirow{4}{*}{1} \\
\hline & 4 & & $1.5155149393260654 \mathrm{i}$ & \multirow{3}{*}{-2.511429026296249} & \multirow{3}{*}{$\begin{array}{l}2 \\
8\end{array}$} & & \\
\hline & $\begin{array}{l}3 \\
3\end{array}$ & $0_{4}$ & $\begin{array}{l}0.5 \mathrm{i} \\
-0.5 \mathrm{i}\end{array}$ & & & \multirow[t]{2}{*}{4} & \\
\hline & -4 & & $-1.5155149393260654 \mathrm{i}$ & & & & \\
\hline
\end{tabular}

TABLE VIII: Singular Bethe roots for $N=12, M=5$. There is one solution with one 1 -string and one 4-string 
TABLE IX: Singular Bethe roots for $N=12, M=5$. There is one solution with one 2 -string and one 3 -string

\begin{tabular}{|c|c|c|c|c|c|c|}
\hline & $J$ & $I_{n}$ & $\lambda$ & $E$ & \multicolumn{2}{|c|}{ Rigged Configuration } \\
\hline \multirow{5}{*}{1.} & 3 & $0_{2}$ & $0.5 \mathrm{i}$ & \multirow{5}{*}{-1.6660659592344356} & \multirow{5}{*}{$\begin{array}{l}2 \\
4\end{array}$} & \multirow{5}{*}{1} \\
\hline & 3 & $\sigma_{2}$ & $-0.5 \mathrm{i}$ & & & \\
\hline & 0 & & 0.0 & & & \\
\hline & 5 & $0_{3}$ & $0.9998311128556481 \mathrm{i}$ & & & \\
\hline & -5 & & $-0.9998311128556481 \mathrm{i}$ & & & \\
\hline
\end{tabular}

TABLE X: Singular Bethe roots for $N=12, M=6$. There are three solutions with four 1-strings and one 2-string

\begin{tabular}{|c|c|c|c|c|c|c|c|}
\hline & $J$ & $I_{n}$ & $\lambda$ & $E$ & \multicolumn{3}{|c|}{ Rigged Configuration } \\
\hline \multirow{6}{*}{1.} & \multirow{6}{*}{$\begin{array}{r}-5 / 2 \\
-3 / 2 \\
3 / 2 \\
5 / 2 \\
5 / 2 \\
7 / 2\end{array}$} & \multirow{4}{*}{$\begin{array}{r}-5 / 2_{1} \\
-3 / 2_{1} \\
3 / 2_{1} \\
5 / 2_{1}\end{array}$} & \multirow{2}{*}{$\begin{array}{l}-0.6905065538178187 \\
-0.29326445955096875\end{array}$} & \multirow{6}{*}{-5.352050317651034} & 0 & & \multirow[t]{6}{*}{0} \\
\hline & & & & & \multirow{2}{*}{2} & \multirow{2}{*}{0} & \\
\hline & & & 0.29326445955096875 & & & & \\
\hline & & & \multirow{3}{*}{$\begin{array}{l}0.6905065538178187 \\
0.5 \mathrm{i} \\
-0.5 \mathrm{i}\end{array}$} & & 2 & 0 & \\
\hline & & \multirow{2}{*}{$0_{2}$} & & & 2 & 2 & \\
\hline & & & & & 2 & 2 & \\
\hline \multirow{5}{*}{2.} & \multirow{5}{*}{$\begin{array}{r}-5 / 2 \\
-1 / 2 \\
1 / 2 \\
5 / 2 \\
5 / 2 \\
7 / 2\end{array}$} & \multirow{3}{*}{$\begin{array}{r}-5 / 2_{1} \\
-1 / 2_{1} \\
1 / 2_{1} \\
5 / 2_{1}\end{array}$} & \multirow{2}{*}{$\begin{array}{l}-0.7003461585874278 \\
-0.08830964234306034\end{array}$} & \multirow{5}{*}{-6.229463841147066} & 0 & & \multirow[t]{5}{*}{0} \\
\hline & & & & & 2 & 0 & \\
\hline & & & $\begin{array}{l}0.08830904234300034 \\
0.7003461585874278\end{array}$ & & 2 & 1 & \\
\hline & & \multirow{2}{*}{$0_{2}$} & \multirow{2}{*}{$\begin{array}{l}0.5 \mathrm{i} \\
-0.5 \mathrm{i}\end{array}$} & & 2 & 1 & \\
\hline & & & & & 2 & 2 & \\
\hline \multirow{6}{*}{3.} & $-3 / 2$ & $-3 / 2_{1}$ & -0.30694160344558236 & & 0 & & \multirow[t]{6}{*}{0} \\
\hline & $-1 / 2$ & $-1 / 2_{1}$ & -0.09083103807287891 & \multirow{5}{*}{-7.77738933370129} & 2 & 1 & \\
\hline & $1 / 2$ & $1 / 2_{1}$ & 0.09083103807287891 & & & & \\
\hline & $3 / 2$ & $3 / 2_{1}$ & 0.30694160344558236 & & 2 & 1 & \\
\hline & \multirow{2}{*}{$7 / 2$} & \multirow[t]{2}{*}{$0_{2}$} & $0.5 \mathrm{i}$ & & 2 & 1 & \\
\hline & & & $-0.5 \mathrm{i}$ & & 2 & 1 & \\
\hline
\end{tabular}

the Bethe eigenstate (5) takes a simple form [16, 21]

$$
|\Psi\rangle_{2} \equiv \sum_{j=1}^{N}(-1)^{j} S_{j}^{-} S_{j+1}^{-}|\Omega\rangle .
$$

Based on (18) and the self-conjugacy condition we can classify the different singular states for a fixed number of down spins. For $M=2$, the only singular solution is of the form

$$
\left\{\frac{i}{2},-\frac{i}{2}\right\}
$$

which is given for $N=12$ in table III. For $M=3$ the only singular solution possible is

$$
\left\{\frac{i}{2},-\frac{i}{2}, 0\right\}
$$

which is given in table IV. Note that for $M=2$ and $M=3$, there is only one singular state for any even $N \geq 4$. For $M=4$, there are two different classes of singular solutions

$$
\begin{array}{ll}
\left\{\frac{i}{2},-\frac{i}{2}, a_{1},-a_{1}\right\} & \text { for } \quad a_{1} \in \mathbb{R}, \\
\left\{\frac{i}{2},-\frac{i}{2}, i a_{1},-i a_{1}\right\} \quad \text { for } \quad a_{1} \in \mathbb{R} .
\end{array}
$$

In table $\mathrm{V}$ we give the first type of solutions and in table VI the second type of solutions. By substituting the singular 
TABLE XI: Singular Bethe roots for $N=12, M=6$. There are four solutions with two 1-strings and one 4-string

\begin{tabular}{|c|c|c|c|c|c|c|c|}
\hline \multirow{2}{*}{\multicolumn{2}{|c|}{$\begin{array}{r}J \\
-5 / 2\end{array}$}} & $I_{n}$ & $\lambda$ & $E$ & \multicolumn{3}{|c|}{ Rigged Configuration } \\
\hline & & $-7 / 2_{1}$ & -0.9468899269636574 & & & & \\
\hline & $5 / 2$ & $7 / 2_{1}$ & 0.9468899269636574 & & 0 & & 0 \\
\hline \multirow{3}{*}{1.} & $9 / 2$ & & $1.5202343808518264 \mathrm{i}$ & -1.3869684651709449 & 6 & 0 & \\
\hline & $5 / 2$ & $0_{1}$ & $0.5 \mathrm{i}$ & & & & \\
\hline & $7 / 2$ & & $-0.5 \mathrm{i}$ & & 0 & 0 & \\
\hline & $-9 / 2$ & & & & & & \\
\hline \multirow{5}{*}{2.} & $-3 / 2$ & $-5 / 2_{1}$ & -0.4781191178586541 & & & & \\
\hline & $3 / 2$ & $5 / 2_{1}$ & 0.4781191178586541 & & 0 & & 0 \\
\hline & $9 / 2$ & & $1.547617992727387 \mathrm{i}$ & -2.623262633690177 & 6 & 1 & \\
\hline & $\begin{array}{l}5 / 2 \\
7 / 2\end{array}$ & $0_{4}$ & 0.51 & & 6 & 5 & \\
\hline & $-9 / 2$ & & $-1.547617992727387 \mathrm{i}$ & & & & \\
\hline \multirow{5}{*}{3.} & $-1 / 2$ & $-3 / 2_{1}$ & -0.24554120504274426 & \multirow{5}{*}{-3.773151385638714} & & & \\
\hline & $1 / 2$ & $3 / 2_{1}$ & 0.24554120504274426 & & 0 & & 0 \\
\hline & $9 / 2$ & & $1.5729034567782043 \mathrm{i}$ & & 6 & 2 & \\
\hline & $7 / 2$ & $0_{4}$ & $-0.5 \mathrm{i}$ & & 6 & 4 & \\
\hline & $-9 / 2$ & & $-1.5729034567782043 \mathrm{i}$ & & & & \\
\hline \multirow{5}{*}{4.} & $1 / 2$ & $-1 / 2_{1}$ & -0.07666936301920817 & \multirow{5}{*}{-4.46706634996716} & & & \multirow{5}{*}{0} \\
\hline & $-1 / 2$ & $1 / 2_{1}$ & 0.07666936301920817 & & 0 & & \\
\hline & $\begin{array}{l}9 / 2 \\
5 / 2\end{array}$ & & $1.5866164135168153 \mathrm{i}$ & & 6 & 3 & \\
\hline & $7 / 2$ & $0_{4}$ & $\begin{array}{l}0.5 \mathrm{l} \\
-0.5 \mathrm{i}\end{array}$ & & 6 & 3 & \\
\hline & $-9 / 2$ & & $-1.5866164135168153 \mathrm{i}$ & & & & \\
\hline
\end{tabular}

TABLE XII: Singular Bethe roots for $N=12, M=6$. There is one solution with one 1-string, one 2-string and one 3 -string

\begin{tabular}{|c|c|c|c|c|c|c|c|}
\hline & $J$ & $I_{n}$ & $\lambda$ & $\bar{E}$ & \multicolumn{3}{|c|}{ Rigged Configuration } \\
\hline & $-1 / 2$ & $\overline{0_{1}}$ & $0.018539899905903653 \mathrm{i}$ & & & & \\
\hline \multirow{4}{*}{1.} & $5 / 2$ & $0_{0}$ & $0.5 \mathrm{i}$ & \multirow{4}{*}{-3.649738189247195} & 0 & & \multirow[t]{4}{*}{0} \\
\hline & $\begin{array}{l}7 / 2 \\
9 / 2\end{array}$ & $\mathrm{U}_{2}$ & $\begin{array}{l}-0.5 \mathrm{i} \\
0.9937750058754778 \mathrm{i}\end{array}$ & & 2 & & \\
\hline & $1 / 2$ & $0_{3}$ & $-0.018539899905903653 \mathrm{i}$ & & 6 & 3 & \\
\hline & $-9 / 2$ & & $-0.9937750058754778 \mathrm{i}$ & & & & \\
\hline
\end{tabular}

roots 22 or 23 in the Bethe ansatz equations (6) we obtain a polynomial equation for a single variable $x$

$$
\left(\frac{x-i}{x+i}\right)^{N-2}=\frac{x-3 i}{x+3 i}, \text { for } \quad N=8,10,12, \cdots,
$$

where $x$ is either $2 a_{1}$ or $2 i a_{1}$, of eq. (22) and (23), respectively. This is a polynomial equation of degree $N-2$ which can be seen from the simplified form

$$
f(x)=A_{N-2} x^{N-2}+A_{N-4} x^{N-4}+\cdots+A_{N-2-2 r} x^{N-2-2 r}+\cdots+A_{0}=0,
$$

where the coefficients $A_{N-2}={ }^{N-2} C_{1}-3, A_{N-4}=-{ }^{N-2} C_{3}+3{ }^{N-2} C_{2}, \cdots, A_{0}=-3(-1)^{(N-2) / 2}$ are all real. It is useful to write the general form of the coefficients as

$$
A_{N-2-2 r}=(-1)^{r}\left[{ }^{N-2} C_{2 r+1}-3^{N-2} C_{2 r}\right], \quad \text { for } \quad r=0,1,2, \cdots,(N-2) / 2 .
$$

It is manifest that if $x$ is a root of the polynomial then $-x$ is also a root, which accounts for the two rapidities of $M=4$ singular solution in (22) and (23). According to the fundamental theorem of algebra the polynomial eq. (25) 
TABLE XIII: Singular Bethe roots for $N=12, M=6$. There is one solution with three 2-strings

\begin{tabular}{|c|c|c|c|c|c|c|}
\hline & $J$ & $I_{n}$ & $\lambda$ & $E$ & \multicolumn{2}{|c|}{ Rigged Configuration } \\
\hline & $-7 / 2$ & & $-0.6620239184153354+0.5045174233098804 \mathrm{i}$ & & & \\
\hline \multirow{4}{*}{1.} & $-5 / 2$ & $-1_{2}$ & $-0.6620239184153354-0.5045174233098804 \mathrm{i}$ & & 0 & 0 \\
\hline & $\begin{array}{l}5 / 2 \\
7 / 2\end{array}$ & $\mathrm{O}_{2}$ & $\begin{array}{l}0.5 \mathrm{i} \\
-0.5 \mathrm{i}\end{array}$ & -2.367482833109191 & 0 & 0 \\
\hline & $5 / 2$ & & $0.6620239184153354+0.5045174233098804 \mathrm{i}$ & & 0 & 0 \\
\hline & $7 / 2$ & $1_{2}$ & $0.6620239184153354-0.5045174233098804 \mathrm{i}$ & & & \\
\hline
\end{tabular}

TABLE XIV: Singular Bethe roots for $N=12, M=6$. There is one solution with one 6 -string

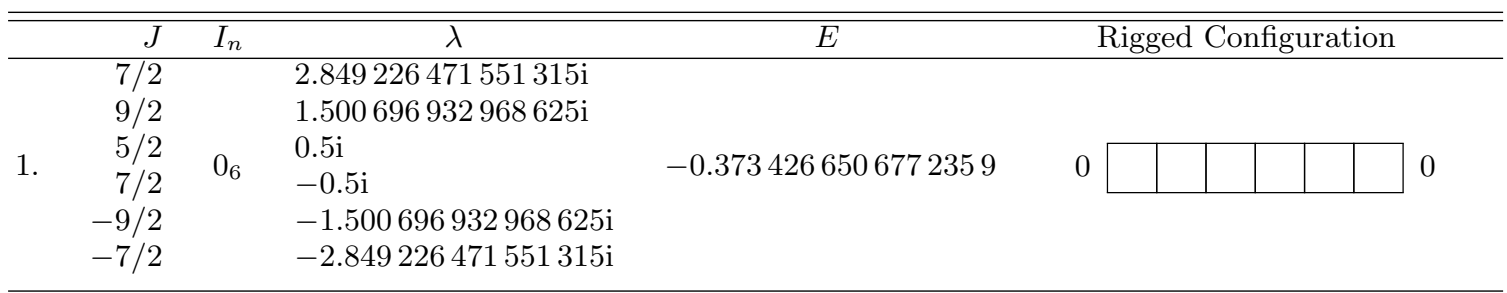

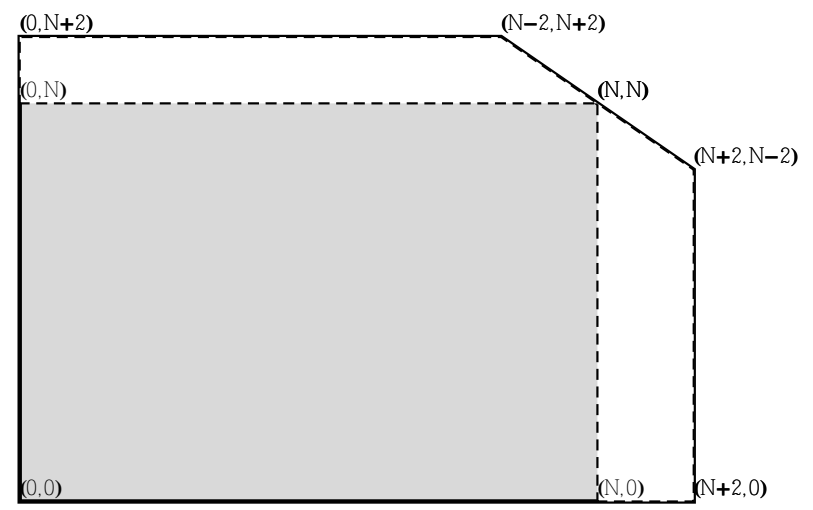

FIG. 3: The Newton polygons of two polynomials $F(x, y)$ and $G(x, y)$ and their Minkowski sum is shown. The area of the the three regions are Area $(\operatorname{New}(F(x, y) \cdot G(x, y)))=N(N+4)-4$, Area $(\operatorname{New}(F(x, y)))=2(N-1)$ and Area $($ New $(G(x, y)))=2(N-1)$ and the mixed area $N^{2}$ is given by the gray square.

has at most $N-2$ distinct roots (real or complex) and since the coefficients are all real the complex roots will occur in complex conjugate pairs if there are any. Considering the fact that two roots of opposite signs of (25) constitute one singular root we find that the total number of singular roots $\mathcal{N}$ for $M=4$ is at most

$$
\mathcal{N}=\frac{N-2}{2}, \quad \text { for } \quad N=8,10,12, \cdots .
$$

One test which guarantees that the the total number of singular solutions is exactly given by 27 is to show that the discriminant of the polynomial $f(x)$ in 25$)$ is not zero, which we cannot prove here. But numerical check for many different values of the chain lengths shows that discriminants are indeed non-zero and negative, it follows that the roots are all distinct and all account for the singular solutions. The number of sign changes $\mathcal{V}_{+}$of the coefficients (26) of $f(x)$ and the number of sign changes $\mathcal{V}_{-}$of the corresponding coefficient of $f(-x)$ are the same and given by

$$
\mathcal{V}_{ \pm}=\frac{N-2}{2}-1, \quad \text { for } \quad N=8,10,12, \cdots .
$$

Then, according to the Descartes' rule of sign, the number of real positive roots $n_{+}$and and the number of real negative roots $n_{-}$are bounded by

$$
n_{ \pm} \leq \mathcal{V}_{ \pm}
$$

where the upper and lower sign of the suffix of left hand side should be considered with the upper and lower sign of the suffix of right hand side, respectively. Eq. 29 implies that there are at most $(N-2) / 2-1$ number of solutions 
of the type (22) and therefore at least 1 solution of the type 23 . Here and for $M=5$ case bellow we assume that the complex root of (25) are all pure imaginary, for which we do not have any analytical proof but it is supported by numerical observations up to $N=500$. So far our numerical solutions shows that there is exactly $(N-2) / 2-1$ number of solutions of the first type and only 1 solution of the second type as can be seen from FIG. 1 obtained for $N=500$.

In a similar fashion singular solutions for $M=5$ can be obtained, where there exist two different types of singular solutions

$$
\begin{aligned}
& \left\{\frac{i}{2},-\frac{i}{2}, 0, a_{1},-a_{1}\right\} \quad \text { for } \quad a_{1} \in \mathbb{R}, \\
& \left\{\frac{i}{2},-\frac{i}{2}, 0, i a_{1},-i a_{1}\right\} \quad \text { for } \quad a_{1} \in \mathbb{R} .
\end{aligned}
$$

In table VII we give the first type of solutions and in table VIII and IX the second type of solutions. One can again substitute 30 or 31 in the Bethe ansatz equations (6) to obtain a polynomial equation for a single variable $x=2 a_{1}$ or $2 a_{1} i$

$$
\left(\frac{x-i}{x+i}\right)^{N-2}=\frac{x-3 i}{x+3 i} \times \frac{x-2 i}{x+2 i}, \quad \text { for } \quad N=10,12,14, \cdots
$$

Note that $x=0$ is a trivial solution of this equation which is not a Bethe roots. So, after factoring out $x$ from 32 we again obtain a polynomial equation of the form (25) but now the coefficients are given by

$$
B_{N-2-2 r}=(-1)^{r}\left[{ }^{N-2} C_{2 r+1}+6^{N-2} C_{2 r-1}-5^{N-2} C_{2 r}\right], \quad \text { for } \quad r=0,1,2, \cdots,(N-2) / 2 .
$$

We can show that the number of sign changes $\mathcal{V}_{+}$of the coefficients 33 of $f(x)$ and the number of sign changes $\mathcal{V}_{-}$ of the corresponding coefficient of $f(-x)$ are the same

$$
\mathcal{V}_{ \pm}=\frac{N-2}{2}-2, \quad \text { for } \quad N=10,12,14, \cdots
$$

Applying the Descartes' rule (29) now implies that there are at most $(N-2) / 2-2$ roots of the form $(30)$ and at least 2 roots of the form (31) making the total number of singular solutions

$$
\mathcal{N}=\frac{N-2}{2}, \quad \text { for } \quad N=10,12,14, \cdots
$$

In FIG. 2, we see that for $N=500, M=5$, there are only two solutions of the type (31) and the rest are of the form (30) and we also checked up to $N=500$ but find no exceptions. For $M=6$, the following four different classes of singular solutions may exist

$$
\begin{aligned}
& \left\{\frac{i}{2},-\frac{i}{2}, a_{1},-a_{1}, a_{2},-a_{2}\right\} \quad \text { for } \quad a_{1}, a_{2} \in \mathbb{R}, \\
& \left\{\frac{i}{2},-\frac{i}{2}, a_{1},-a_{1}, i a_{2},-i a_{2}\right\} \quad \text { for } \quad a_{1}, a_{2} \in \mathbb{R}, \\
& \left\{\frac{i}{2},-\frac{i}{2}, i a_{1},-i a_{1}, i a_{2},-i a_{2}\right\} \quad \text { for } \quad a_{1}, a_{2} \in \mathbb{R}, \\
& \left\{\frac{i}{2},-\frac{i}{2}, a_{1} \pm i a_{2},-a_{1} \pm i a_{2}\right\} \quad \text { for } \quad a_{1}, a_{2} \in \mathbb{R} .
\end{aligned}
$$

In table X solutions of the form (36), in Table XI solutions of the form (37), in table XII and table XIV solutions of the form (38), and in Table XIII solutions of the form $\sqrt{39}$ have been displayed. A system of two variable $(x, y)$ polynomial equations for even $N \geq 12, M=6$ can be obtained by substituting any of the form of the roots 36 - (39) in the Bethe Ansatz equations $(6)$ as

$$
\begin{aligned}
& \left(\frac{x-i}{x+i}\right)^{N-2}=\frac{x-3 i}{x+3 i} \times \frac{x-y-2 i}{x-y+2 i} \times \frac{x+y-2 i}{x+y+2 i}, \\
& \left(\frac{y-i}{y+i}\right)^{N-2}=\frac{y-3 i}{y+3 i} \times \frac{y-x-2 i}{y-x+2 i} \times \frac{y+x-2 i}{y+x+2 i},
\end{aligned}
$$


which can be rewritten as

$$
\begin{aligned}
F(x, y)= & \sum_{r=0}^{\frac{N-2}{2}}(-1)^{r}\left[\left({ }^{N-2} C_{2 r+1}-{ }^{N-2} C_{2 r}\right) x^{N-2 r}+\left(8^{N-2} C_{2 r+1}-12^{N-2} C_{2 r}\right) x^{N-2-2 r}\right. \\
& \left.-\left({ }^{N-2} C_{2 r+1}+3^{N-2} C_{2 r}\right) x^{N-2-2 r} y^{2}\right]=0 \\
G(x, y)= & \sum_{r=0}^{\frac{N-2}{2}}(-1)^{r}\left[\left({ }^{N-2} C_{2 r+1}-{ }^{N-2} C_{2 r}\right) y^{N-2 r}+\left(8^{N-2} C_{2 r+1}-12^{N-2} C_{2 r}\right) y^{N-2-2 r}\right. \\
& \left.-\left({ }^{N-2} C_{2 r+1}+3^{N-2} C_{2 r}\right) y^{N-2-2 r} x^{2}\right]=0 .
\end{aligned}
$$

where the pair $(x, y)$ can either be $\left(2 a_{1}, 2 a_{2}\right),\left(2 a_{1}, 2 i a_{2}\right),\left(2 i a_{1}, 2 i a_{2}\right),\left(2 a_{1}+2 i a_{2}, 2 a_{1}-2 i a_{2}\right)$ or $\left(2 a_{1}+2 i a_{2},-2 a_{1}+2 i a_{2}\right)$. Note that the two equations (42) are symmetric with respect to the permutations of the variables. Although solutions of this system of equations give all the desired roots of the form (36) - (39), they also give roots such as $x= \pm y$ and $x \neq \pm y^{*}(\mathbb{R}(x)=\mathbb{R}(y) \neq 0)$ which are not physical solutions and therefore should be discarded. In order to calculate the number of singular solutions we have to first find out the number of solutions of $(42)$ and subtract the number of solutions of type $x= \pm y$ and $x \neq \pm y^{*}(\mathbb{R}(x)=\mathbb{R}(y) \neq 0)$. According to Bernstein's theorem 34 number of solutions of a system of generic polynomial equations of two variables of the form $f_{1}(x, y)=0, f_{2}(x, y)=0$ in $(\mathbb{C} \backslash 0)^{2}$ is given by their mixed area $\mathcal{M}\left(\operatorname{New}\left(f_{1}\right), \operatorname{New}\left(f_{2}\right)\right)$, where $\operatorname{New}\left(f_{1}\right)$ and $\operatorname{New}\left(f_{2}\right)$ are the Newton polygons of $f_{1}(x, y)$ and $f_{2}(x, y)$, respectively. By inspecting (42) we can readily obtain the Newton polygons New $(F(x, y))$ and New $(G(x, y))$ of the system of equations as

$$
\begin{aligned}
& \text { New }(F(x, y)):=\operatorname{conv}\{(N, 0),(N-2,0), \cdots,(0,0),(N-2,2),(N-4,2), \cdots,(0,2)\}, \\
& \text { New }(G(x, y)):=\operatorname{conv}\{(0, N),(0, N-2), \cdots,(0,0),(2, N-2),(2, N-4), \cdots,(2,0)\} .
\end{aligned}
$$

The Minkowski sum New $(F(x, y) \cdot G(x, y))$ of the two polygons can be obtained from the multiplication of the corresponding polynomials and can be written as

$$
\begin{array}{cccccccc}
\text { New }(F(x, y) \cdot G(x, y)):= \\
\begin{array}{cccccccc}
\operatorname{conv}\{(N, N), & (N-2, N), & \cdots, & (0, N), & (N-2, N+2), & (N-4, N+2), & \cdots, & (0, N+2), \\
(N, N-2), & (N-2, N-2), & \cdots, & (0, N-2), & (N-2, N), & (N-4, N), & \cdots, & (0, N), \\
\vdots & \vdots & \ddots & \vdots & \vdots & \vdots & \ddots & \vdots \\
(N, 0), & (N-2,0), & \cdots, & (0,0), & (N-2,2), & (N-4,2), & \cdots, & (0,2), \\
(N+2, N-2), & (N, N-2), & \cdots, & (2, N-2), & (N, N), & (N-2, N), & \cdots, & (2, N), \\
(N+2, N-4), & (N, N-4), & \cdots, & (2, N-4), & (N, N-2), & (N-2, N-2), & \cdots, & (2, N-2), \\
\vdots & \vdots & \ddots & \vdots & \vdots & \vdots & \ddots & \vdots \\
(N+2,0), & (N, 0), & \cdots, & (2,0), & (N, 2), & (N-2,2), & \cdots, & (2,2)\} .
\end{array}
\end{array}
$$

Now one can obtain the mixed area

$$
\mathcal{M}(\operatorname{New}(F) \cdot \text { New }(G))=\text { Area }(\operatorname{New}(F(x, y) \cdot G(x, y)))-\operatorname{Area}(\operatorname{New}(F(x, y)))-\operatorname{Area}(\operatorname{New}(G(x, y)))=N^{2},
$$

which is the area of the gray colored square in FIG. 3. There are $2(N-2)$ solutions of the form $x= \pm y$, which can be shown analytically easily because in this case we can reduce the Bethe ansatz equation to an one variable polynomial equation of degree $(N-2)$. From the numerical observation for $N=12,14,16$ we see that there are $4(N-1)$ roots which are of the form $x \neq \pm y^{*}(\mathbb{R}(x)=\mathbb{R}(y) \neq 0)$ and we assume this to be valid for any $N \geq 12$. For $N=16, M=6,7$, singular solutions have been shown in Table XV, XVI respectively. So the total number of singular solutions becomes

$$
\mathcal{N}=\frac{1}{8}\left(N^{2}-6 N+8\right) \quad \text { for } \quad N=12,14, \cdots
$$

The overall factor 8 in the denominator is the multiplicity of the singular roots. Note that two roots of 42 are considered the same if upon substitution in (36)- (39) gives the same singular roots. Similarly, $M=7$ case can also 
be discussed, where there are the following four different class of singular solutions

$$
\begin{aligned}
& \left\{\frac{i}{2},-\frac{i}{2}, 0, a_{1},-a_{1}, a_{2},-a_{2}\right\} \quad \text { for } \quad a_{1}, a_{2} \in \mathbb{R}, \\
& \left\{\frac{i}{2},-\frac{i}{2}, 0, a_{1},-a_{1}, i a_{2},-i a_{2}\right\} \quad \text { for } \quad a_{1}, a_{2} \in \mathbb{R}, \\
& \left\{\frac{i}{2},-\frac{i}{2}, 0, i a_{1},-i a_{1}, i a_{2},-i a_{2}\right\} \quad \text { for } \quad a_{1}, a_{2} \in \mathbb{R}, \\
& \left\{\frac{i}{2},-\frac{i}{2}, 0, a_{1} \pm i a_{2},-a_{1} \pm i a_{2}\right\} \quad \text { for } \quad a_{1}, a_{2} \in \mathbb{R} .
\end{aligned}
$$

A system of two variable $(x, y)$ polynomial equations can be obtained by substituting any of the form of the roots (47)- 50 in the Bethe Ansatz equations (6) as

$$
\begin{aligned}
& \left(\frac{x-i}{x+i}\right)^{N-2}=\frac{x-3 i}{x+3 i} \times \frac{x-2 i}{x+2 i} \times \frac{x-y-2 i}{x-y+2 i} \times \frac{x+y-2 i}{x+y+2 i} \\
& \left(\frac{y-i}{y+i}\right)^{N-2}=\frac{y-3 i}{y+3 i} \times \frac{y-2 i}{y+2 i} \times \frac{y-x-2 i}{y-x+2 i} \times \frac{y+x-2 i}{y+x+2 i}
\end{aligned}
$$

where $(x, y)$ has been defined in eq. (42). The number of singular solutions is given by the the formula 46 but now $N=14,16, \cdots$. Generally, for even- $N$ and even- $M$, the singular root looks like

$$
\begin{aligned}
& \left\{ \pm \frac{i}{2}, \pm a_{1}, \pm a_{2}, \cdots, \pm a_{n_{1}}, \pm i b_{1}, \pm i b_{2}, \cdots, \pm i b_{n_{2}}, \pm c_{1} \pm i d_{1}, \pm c_{2} \pm i d_{2}, \cdots, \pm c_{n_{3}} \pm i d_{n_{3}}\right\} \\
& \text { for } \quad a_{i}, b_{i}, c_{i}, d_{i} \in \mathbb{R} ; n_{1}, n_{2} \in\left[0,1,2, \cdots, \frac{M-2}{2}\right] ; n_{3} \in\left[0,1,2, \cdots, \frac{M-2}{4}\right] ; 2 n_{1}+2 n_{2}+4 n_{3}=M-2,
\end{aligned}
$$

and for even- $N$ and odd $M$, the singular root looks like

$$
\begin{aligned}
& \left\{ \pm \frac{i}{2}, 0, \pm a_{1}, \pm a_{2}, \cdots, \pm a_{n_{1}}, \pm i b_{1}, \pm i b_{2}, \cdots, \pm i b_{n_{2}}, \pm c_{1} \pm i d_{1}, \pm c_{2} \pm i d_{2}, \cdots, \pm c_{n_{3}} \pm i d_{n_{3}}\right\} \\
& \text { for } \quad a_{i}, b_{i}, c_{i}, d_{i} \in \mathbb{R} ; n_{1}, n_{2} \in\left[0,1,2, \cdots, \frac{M-3}{2}\right] ; n_{3} \in\left[0,1,2, \cdots, \frac{M-3}{4}\right] ; 2 n_{1}+2 n_{2}+4 n_{3}=M-3 .
\end{aligned}
$$

Solving this problem for the number of singular solutions becomes complicated as $M$ increases but a general form of the number of singular solutions can be written as

$$
\mathcal{N}=\frac{1}{p_{0}}\left(N^{n}+p_{1} N^{n-1}+p_{2} N^{n-2}+\cdots+p_{n}\right) \quad \text { for } \quad \text { even } N \geq 2 M
$$

where the integer $p_{0}$ is the multiplicity of the singular roots, $p_{i}$ 's are some integers and for even $M, n=(M-2) / 2$, or for odd $M, n=(M-3) / 2$. From the analysis of singular solutions up to $M=7$ we conjecture that for even $N$ the number of singular solutions for even $M$ and $M+1$ are same.

\section{RIGGED CONFIGURATIONS}

It has been observed that there is a connection between the Bethe states and the rigged configurations 35] 37. It offers a nice bijection between the Bethe states and the rigged configurations at least for a not very long spin-1/2 chain. In $N=12$ case of the isotropic spin-1/2 chain, we establish this bijection for the singular solutions and for the non self-conjugate string solutions comparing their Takahashi quantum numbers with the riggings of a rigged configuration.

To understand what a rigged configuration is and how it works let us give here a brief account of the basic idea behind the rigged configurations. We keep the notations of [35. This is a Young Tableau like object with two sets of integers, one in the left hand side of the boxes known as vacancy numbers $P_{k}(\nu)$, and the other on the right hand side of the boxes known as riggings $J_{k, \alpha}$. Consider an eigenstate of the Hamiltonian of a spin-1/2 chain of length $N$ and total $M$ down-spins in the state. The down-spins can be partitioned in different ways and each partition can be written as $\nu=\left\{\nu_{1}, \nu_{2}, \cdots, \nu_{s}\right\}$ such that the parts $\nu_{i}$ 's are positive integers and $s$ is the total number of parts in a particular partition. Since all the down-spins have been partitioned in $\nu$ it satisfies $\sum_{i=1}^{s} \nu_{i}=M$. In 
the string-solution language, for example, $M=9$ down-spins with two 3 -strings, one 2-string and one 1-string has a partition $\nu=\{3,3,2,1\}$. The set of vacancy numbers $P_{k}(\nu)$ which need to be all non-negative in order to have a viable configuration are defined for a spin- $1 / 2$ system as follows

$$
P_{k}(\nu)=N-2 \sum_{i=1}^{s} \min \left(k, \nu_{i}\right),
$$

where $k=1,2, \cdots$ is the length of a string under consideration. Once a vacancy number is obtained then one can get a bound for the set of corresponding riggings $J_{k, \alpha}$ as

$$
0 \leq J_{k, 1} \leq J_{k, 2} \leq \cdots \leq J_{k, M_{k}} \leq P_{k}(\nu)
$$

where $M_{k}$ is the total number of $k$-strings in a particular set of roots defining a state. In order to have a bijection between the rigged configurations and the Bethe states we need to define a flip map $\kappa$ as

$$
\kappa\left(J_{k, \alpha}\right)=P_{k}(\nu)-J_{k, M_{k}-\alpha+1}
$$

A rigged configuration of the form $(\nu, J)$ therefore have two different classes of configurations, one which are flipinvariant and the other which are not flip-invariant under the transformation (58).

Given a partition $\nu$ and a set of corresponding Bethe states it is now our task to assign a rigged configuration $(\nu, J)$ to a Bethe root. One way to assign this is to compare between the riggings $J$ and the real part of the rapidities of Bethe roots and assign higher value of the real part of the roots to higher value of the riggings as adopted in 35 . To get a mapping based on the comparison with the rapidity we have to then actually solve the rapidities numerically. We instead considered a comparison between the set of riggings of $k$-length strings $\left\{J_{k, 1}, J_{k, 2}, \cdots, J_{k, M_{k}}\right\}$ in a form of the lexicographical order and the set of Takahashi quantum numbers of the same $k$-length strings $\left\{I_{k}^{1}, I_{k}^{2}, \cdots, I_{k}^{M_{k}}\right\}$ in a form of the inverse lexicographical order. The comparison starts from the largest length strings to the lowest length strings in descending order. We assign larger riggings to larger Takahashi quantum numbers in the order specified in the above and obtain a bijection between the Bethe states and the rigged configurations. On the right hand side of each row of the Tables I to XIV we have shown the corresponding rigged configurations for $N=12$ spin- $1 / 2$ chain.

To understand the mapping, let us consider all solutions of the form of one 2-string and two 1-strings in $N=12, M=4$. Since the largest string in this example is one 2-string, we first compare the Takahashi quantum numbers of the two string $\left\{I_{2}\right\}=\{2,1,0,-1,-2\}$ with the riggings of the 2-string $\left\{J_{2,1}\right\}=\{4,3,2,1,0\}$ from the left to the right. Then we compare 28 pairs of the Takahashi quantum numbers for two 1 -strings, $\left\{I_{1}^{1}, I_{1}^{2}\right\}=$ $\{(7 / 2,5 / 2),(7 / 2,3 / 2), \cdots,(7 / 2,-7 / 2),(5 / 2,3 / 2),(5 / 2,1 / 2), \cdots,(5 / 2,-7 / 2),(3 / 2,1 / 2),(3 / 2,-1 / 2), \cdots,(3 / 2,-7 / 2)$, $(1 / 2,-1 / 2),(1 / 2,-3 / 2),(1 / 2,-5 / 2),(1 / 2,-7 / 2),(-1 / 2,-3 / 2),(-1 / 2,-5 / 2),(-1 / 2,-7 / 2),(-3 / 2,-5 / 2)$, $(-3 / 2,-7 / 2),(-5 / 2,-7 / 2)\}$, with 28 pairs of riggings of the two 1-strings, $\left\{J_{1,1}, J_{1,2}\right\}=\{(6,6),(5,6)$, $\cdots,(0,6),(5,5),(4,5), \cdots,(0,5),(4,4),(3,4), \cdots,(0,4),(3,3),(2,3),(1,3),(0,3),(2,2),(1,2),(0,2),(1,1),(0,1),(0,0)\}$ from the left to the right. Four such explicit correspondences have been given in TABLE V. This is just one example, the mapping for all solutions of the $N=12$ spin chain will be reported elsewhere.

\section{CONCLUSIONS}

We have observed that in the isotropic spin-1/2 Heisenberg model there are some string solutions which do not fall in the standard category of string solutions. These are physical solutions of the Bethe ansatz equations where the central rapidity of some of the odd length strings in a given Bethe state becomes complex contrary to the standard knowledge where the central rapidity of an odd-length string is considered to be real even in the deformed strings. Some of the individual strings in a Bethe state in such scenario are no longer self-conjugate, but collectively all the strings in the Bethe state remain self-conjugate. This behavior starts from $N=12$ case, where we see that in a $M=5$ down spin sector the central rapidity of one of the two 1-strings and the central rapidity of a 3-string become complex conjugate to each other as shown in Table I. In $M=6$ down spin sector with $M_{1}=M_{2}=M_{3}=1$ we also observed that the the central rapidity of an 1-string and the central rapidity of a 3 -string become complex conjugate, which are shown in Table II. To obtain these types of solutions in the string picture we have used the Newton- Raphson method in Mathematica and made use of the roots of Takahashi string with some modifications as the initial guess for the input in the iteration method. The number of missing solutions in the deformed string picture for large $N$ is solely attributed to the collapse of pairs of strings in [20] but as we observe in our analysis that the missing string solutions include not only the collapsing strings but also the non self-conjugate strings. 
TABLE XV: Singular solutions for $N=16, M=6$

\begin{tabular}{lllll}
\hline \hline & $\pm \lambda_{1}$ & \multicolumn{1}{c}{$\pm \lambda_{2}$} & \multicolumn{1}{c}{$\pm \lambda_{3}$} & \multicolumn{1}{c}{$E$} \\
\hline 1. & $\pm 0.5 \mathrm{i}$ & $\pm 2.5503138374817507 \mathrm{i}$ & \pm 1.5000074388001383 & -0.34011048736365523 \\
2. & $\pm 0.5 \mathrm{i}$ & $\pm 1.3796268446813218 \pm 0.5617044948437927 \mathrm{i}$ & $\pm 1.3796268446813218 \mp 0.5617044948437927 \mathrm{i}$ & -1.6359517330815763 \\
3. & $\pm 0.5 \mathrm{i}$ & $\pm 1.5006551279784253 \mathrm{i}$ & \pm 1.4468750235773047 & -0.9272127431773511 \\
4 & $\pm 0.5 \mathrm{i}$ & $\pm 1.5015054809619202 \mathrm{i}$ & \pm 0.7685335795282152 & -1.6906915160727207 \\
5. & $\pm 0.5 \mathrm{i}$ & \pm 1.1888973013271522 & \pm 0.5651518180485309 & -3.3573957459677906 \\
6. & $\pm 0.5 \mathrm{i}$ & $\pm 1.5025003507851975 \mathrm{i}$ & \pm 0.4815333019660799 & -2.577099709955288 \\
7. & $\pm 0.5 \mathrm{i}$ & $\pm 1.5034574565626846 \mathrm{i}$ & \pm 0.3032154126087406 & -3.4270758848318454 \\
8. & $\pm 0.5 \mathrm{i}$ & $\pm 1.5042054296808929 \mathrm{i}$ & \pm 0.16920613581844915 & -4.092118420762521 \\
9. & $\pm 0.5 \mathrm{i}$ & $\pm 1.5046139317879763 \mathrm{i}$ & \pm 0.05454496804382775 & -4.456399292620959 \\
10. & $\pm 0.5 \mathrm{i}$ & \pm 1.2188129982453162 & \pm 0.3397640630514992 & -4.3126312988210485 \\
11. & $\pm 0.5 \mathrm{i}$ & \pm 1.230393777779506 & \pm 0.18594643292085092 & -5.080934412797139 \\
12. & $\pm 0.5 \mathrm{i}$ & $\pm 0.7904601887000694 \pm 0.4978723207970831 \mathrm{i}$ & $\pm 0.7904601887000694 \mp 0.4978723207970831 \mathrm{i}$ & -2.2383133111757774 \\
13. & $\pm 0.5 \mathrm{i}$ & \pm 1.2347813367503913 & \pm 0.059477281275134256 & -5.50766928015777 \\
14. & $\pm 0.5 \mathrm{i}$ & $\pm 0.9998878483722456 \mathrm{i}$ & $\pm 0.0020454192544636087 \mathrm{i}$ & -3.666334749363233 \\
15. & $\pm 0.5 \mathrm{i}$ & \pm 0.6123545410064503 & \pm 0.3525626922450306 & -5.2717066348913075 \\
16. & $\pm 0.5 \mathrm{i}$ & $\pm 0.3690085276994174 \pm 0.5000006776237689 \mathrm{i}$ & $\pm 0.3690085276994174 \mp 0.5000006776237689 \mathrm{i}$ & -2.760293288634319 \\
17. & $\pm 0.5 \mathrm{i}$ & \pm 0.6182552619465121 & \pm 0.19131857038330927 & -6.0708284216665955 \\
18. & $\pm 0.5 \mathrm{i}$ & \pm 0.6205808020482364 & \pm 0.060997149042557576 & -6.515846827650192 \\
19. & $\pm 0.5 \mathrm{i}$ & \pm 0.3614712275510142 & \pm 0.19402031893529728 & -7.103527226422651 \\
20. & $\pm 0.5 \mathrm{i}$ & \pm 0.3626356905570207 & \pm 0.06177909091569003 & -7.561051834047694 \\
21. & $\pm 0.5 \mathrm{i}$ & \pm 0.1958958475087899 & \pm 0.062168651323811226 & -8.406807180538566 \\
\hline
\end{tabular}

TABLE XVI: Singular solutions for $N=16, M=7$

\begin{tabular}{|c|c|c|c|}
\hline$\lambda_{0} \pm \lambda_{1}$ & $\pm \lambda_{2}$ & $\pm \lambda_{3}$ & $E$ \\
\hline 1. $0 . \pm 0.5 \mathrm{i}$ & $\pm 2.6298433893582316 \mathrm{i}$ & $\pm 1.5000914661481464 \mathrm{i}$ & -2.350055306797338 \\
\hline 2. $\quad 0 . \pm 0.5 \mathrm{i}$ & $\pm 2.07424802947408 \mathrm{i}$ & $\pm 0.9999998530014925 \mathrm{i}$ & -1.4199051831445788 \\
\hline 3. $0 . \pm 0.5 \mathrm{i}$ & $\pm 1.4938977252774208 \mathrm{i}$ & $\pm 1.0000016566330572 \mathrm{i}$ & -1.162063053432737 \\
\hline 4. $0 . \pm 0.5 \mathrm{i}$ & $\pm 1.0698188196969034 \pm 0.5269468241303141 \mathrm{i}$ & $\pm 1.0698188196969034 \mp 0.5269468241303141 \mathrm{i}$ & -3.8868975847171865 \\
\hline 5. $\quad 0 . \pm 0.5 \mathrm{i}$ & $\pm 1.5041698213826247 \mathrm{i}$ & \pm 1.221139754130827 & -3.0774346215313986 \\
\hline 6. $0 . \pm 0.5 \mathrm{i}$ & $\pm 1.5093657772817572 \mathrm{i}$ & \pm 0.6592952083776108 & -3.9675056171559397 \\
\hline 7. $0 . \pm 0.5 \mathrm{i}$ & $\pm 1.515015400079057 \mathrm{i}$ & \pm 0.40717599380712116 & -4.91611439705291 \\
\hline 8. $\quad 0 . \pm 0.5 \mathrm{i}$ & $\pm 1.5199350759696575 \mathrm{i}$ & \pm 0.24334683943383273 & -5.748578542605349 \\
\hline 9. $\quad 0 . \pm 0.5 \mathrm{i}$ & $\pm 1.5232105503989217 \mathrm{i}$ & \pm 0.11488010687146354 & -6.316377039659267 \\
\hline 10. $0 . \pm 0.5 \mathrm{i}$ & \pm 1.1253554306955575 & $\pm 0.9999913073015081 \mathrm{i}$ & -2.326081561803587 \\
\hline 11. $0 . \pm 0.5 \mathrm{i}$ & \pm 0.971587089884476 & \pm 0.4785693257863526 & -5.925091964375922 \\
\hline 12. $0 . \pm 0.5 \mathrm{i}$ & \pm 0.9925812662218414 & \pm 0.27359796126615227 & -6.887862445685274 \\
\hline 13. $0 . \pm 0.5 \mathrm{i}$ & \pm 1.0006714145894906 & \pm 0.12688494211216567 & -7.557129929741305 \\
\hline 14. $0 . \pm 0.5 \mathrm{i}$ & $\pm 0.9999742359954078 \mathrm{i}$ & \pm 0.594176313334094 & -3.3248247477794965 \\
\hline 15. $0 . \pm 0.5 \mathrm{i}$ & $\pm 0.9999307341856244 \mathrm{i}$ & \pm 0.35255560825562576 & -4.338106532666103 \\
\hline 16. $0 . \pm 0.5 \mathrm{i}$ & $\pm 0.999771273297567 \mathrm{i}$ & \pm 0.19159560709486184 & -5.15371153919209 \\
\hline 17. $0 . \pm 0.5 \mathrm{i}$ & $\pm 0.9977671372001798 \mathrm{i}$ & \pm 0.05965855699449792 & -5.602542059539793 \\
\hline 18. $0 . \pm 0.5 \mathrm{i}$ & $\pm 0.5060655103518128 \pm 0.49993998176856996 \mathrm{i}$ & $\pm 0.5060655103518128 \mp 0.49993998176856996 \mathrm{i}$ & i-4.592752302306033 \\
\hline 19. $0 . \pm 0.5 \mathrm{i}$ & \pm 0.508511716004234 & \pm 0.28203642842527493 & -8.00073400771648 \\
\hline 20. $0 . \pm 0.5 \mathrm{i}$ & \pm 0.5120886445583803 & \pm 0.13008644102655006 & -8.698635838944963 \\
\hline 21. $0 . \pm 0.5 \mathrm{i}$ & \pm 0.28740641115499543 & \pm 0.131559767036299 & -9.747595724152248 \\
\hline
\end{tabular}

Note that if one allows large deviations then the non self-conjugate strings, which we investigated in this paper, can always be arranged in such a way that the individual strings become self-conjugate. For example, any complex solution can be written in terms of combination of 1-strings and 2-strings, because complex rapidities always appear in complex conjugate pairs. In some cases, it is even possible to arrange the non self-conjugate string solutions in terms of self-conjugate strings involving larger than 2-strings. However we instead opted for non self-conjugate string arrangement for the following reason: We constructed all the solutions as small deformations of the pure string solutions so that we can use the roots of the pure string solutions as an initial guess in the iteration process. If we introduce the pure string solutions as the initial guess, it is relatively easy to find out the small deviations in the iteration method as far as we have investigated. In the string hypothesis in the limit that the small deviations vanish, we obtain the Takahashi quantum numbers, which have unique one-to-one correspondence with the not collapsing string solutions. 
In our examples of non self-conjugate string solutions mentioned in Tables I and II we emphasize this unique correspondence of the Takahashi quantum numbers ( shown in the third column from the left ) to the non self-conjugate string solutions. Just to clarify the point, let us consider the first solution of Table I, which is composed of one real 1string (0.18071431863183055), one complex 1-string (0.44476350644863927 - 0.01877019940237738i) and one 3-string $(0.49181421369589934+0.9614711323790809 i, 0.44476350644863927+0.01877019940237738 i, 0.49181421369589934-$ $0.9614711323790809 i$ ) with a complex center. Once we take the $\Delta_{\alpha a}^{j} \rightarrow 0$ limit, we recover the Takahashi quantum numbers associated with them, which are also derived directly by making use of eq. (11). On the other hand, if one arranges the above mentioned solution in the form of one 1-string (0.18071431863183055), and two 2-strings $(0.49181421369589934+0.9614711323790809 i, 0.49181421369589934-0.9614711323790809 i)$ and $(0.44476350644863927+0.01877019940237738 i, 0.44476350644863927-0.01877019940237738 i)$ with large deviations, then the self-conjugacy is restored for each individual string, while there is no set of Takahashi quantum numbers available for this arrangement in the string picture. Here we recall that without Takahashi quantum numbers we do not have any good initial guess in the iteration method of finding the solutions to the Bethe ansatz equations, while the solution of the Takahashi equations for a given set of Takahashi quantum numbers leads to a good initial guess as far as we studied. The same is true if one arranges the above solution in the form of one 1string $(0.18071431863183055)$ and one 4 -string $(0.49181421369589934+0.9614711323790809 i, 0.44476350644863927+$ $0.01877019940237738 i, 0.44476350644863927-0.01877019940237738 i, 0.49181421369589934-0.9614711323790809 i)$ with large deviations. Similarly, every non self-conjugate solution we showed in this paper is given in the unique arrangement that is mapped to a set of the Takahashi quantum numbers.

Considering the sum of the rapidities of a singular Bethe state to be zero for even-length spin chains we have classified the singular roots and obtained a general form of the rapidities. For $M=4,5$ it enables us to reduce the Bethe ansatz equations to a polynomial equation of one variable, which can be easily handled numerically for large even $N$ of the spin chain. We showed that for $M=4$ and $M=5$ there are at most $\mathcal{N}=(N-2) / 2$ singular solutions for $N \geq 2 M$. For $M=6,7$ it is possible to reduce the Bethe ansatz equations to a system of polynomial equations of two variables. Making use of the algebraic method we showed that the number of singular solutions in such cases are at most $\mathcal{N}=\left(N^{2}-6 N+8\right) / 8$. For generic values of even $N$ and even $M$ we can not give the number of singular solutions present but we can predict a form of the formula (55) for the number of singular solutions with coefficients

yet to be determined. Our analysis on the number of singular solutions agrees with the value $\frac{N-2}{2} C_{\frac{M-2}{2}}$ for even $M$ and $M+1$, which is one of the conjecture in [35. However, we showed with examples that the number of singular solutions for even- $M$ and $M+1$ are the same at least up to $M=6$, which disagrees with the conjecture 11(C) of [35], and there is no forbidden rigging in connection with the singular solutions in the $N=12$ spin chain. The eigenvalues for the singular states have been obtained by making use of the regularization scheme of [10, 27, 28, 36]. The detail derivation of the eigenvalues and the eigenstates for the singular solutions has been given in [38], where we made use of the same regularization scheme.

\section{ACKNOWLEDGEMENT}

The present study is partially supported by Grant-in-Aid for Scientific Research No. 24540396. P. Giri acknowledges the financial support from JSPS.

[1] H. A. Bethe, Z. Phys. 71 205-226 (1931).

[2] M. Gaudin, "La Fonction d' Onde de Bethe", (Paris: Masson) (1983).

[3] L. D. Faddeev and L. A. Takhtajan, J. Sov. Math. 24241 (1984).

[4] V. Korepin, N. Bogoliubov and A. Izergin, "Quantum Inverse Scattering Method and Correlation Functions", (Cambridge: Cambridge University Press) (1993).

[5] M. Takahashi, "Thermodynamics of One-Dimensional Solvable Models", (Cambridge: Cambridge University Press) (1999).

[6] R. I. Nepomechie, Int. J. Mod. Phys. B 13 2973-2986 (1999).

[7] L. D. Faddeev, Les Houches Summer School Proceedings, 64, Ed. A. Connes, K. Gawedzki and J. Zinn-Justin (Amsterdam: North Holland) 149-219 (1995), (arXiv: hep-th/9605187).

[8] T. Koma and H. Ezawa, Prog. Tehor. Phys. 781009 (1987).

[9] S. N. Martynov, Phys. Lett. A 219 329-334 (1996).

[10] N. Beisert, J. A. Minahan, M. Staudacher and K. Zarembo, JHEP 09010 (2003).

[11] B. Sutherland, "Beautiful Models: 70 Years of Exactly Solved Quantum Many-Body Problems", (Singapore: World Scientific) (2004).

[12] M. Takahashi, Prog. Theor. Phys. 46401 (1971). 
[13] J-S. Caux and J- M. Maillet, Phys. Rev. Lett. 95077201 (2005).

[14] J-S. Caux, R. Hagemans and J- M. Maillet, J. Stat. Mech: Theor. Exp. P09003 (2005).

[15] A. A. Vladimirov, Phys. Lett. A 105 418-420 (1984).

[16] F. H. L. Essler, V. E. Korepin and K. Schoutens, J. Phys. A: Math. Gen. 254115 (1992).

[17] K. Isler and M. B. Paranjape, Phys. Lett. B 319209 (1993).

[18] A. Ilakovac, M. Kolanović, S. Pallua and P. Prester, Phys. Rev. B 607271 (1999).

[19] T Fujita, T. Kobayashi and H. Takahashi, J. Phys. A: Math. Gen. 36 1553-1564 (2003).

[20] R. Hagemans and J-S Caux, J. Phys. A: Math. Theor. 40 14605-14647 (2007).

[21] W. Hao, R. I. Nepomechie and A. J. Sommese, Phy. Rev. E 88052113 (2013).

[22] A. A. Vladimirov, Theor. Math. Phys. 66102 (1986).

[23] F. Woynarovich, J. Phys. A 152985 (1982).

[24] O. Babelon, H. J. de Vega and C. M. Viallet, Nucl. Phys. B 220 13-34 (1983).

[25] K. Fabricius and B. M. McCoy, J. Stat. Phys. 103 647-678 (2001).

[26] K. Fabricius and B. M. McCoy, J. Stat. Phys. 104 573-587 (2001).

[27] R. I. Nepomechie and C. Wang, J. Phys. A: Math. Theor. 46325002 (2013).

[28] L. V. Avdeev and A. A. Vladimirov, Theor. Math. Phys. 691071 (1987).

[29] R. Siddharthan, arXiv:cond-mat/9804210.

[30] A. N. Kirillov and N. Yu. Reshetikhin, J. Sov. Math. 41 925-955 (1988).

[31] A. N. Kirillov, J. Geom. Phys. 5 365-389 (1988).

[32] A. N. Kirillov, St. Petersburg Math. J. 12 161-190 (2001).

[33] T. Deguchi, J. Phys. A Math. Gen. 34 9755-9775 (2001).

[34] B. Sturmfels, "Solving Systems of Polynomial Equations (CBMS Regional Conference Series in Mathematics)", (University of California- AMS|CBMS) (2002).

[35] A. N. Kirillov and R. Sakamoto, J. Phys. A. Math. Theor. 47205207 (2014).

[36] A. N. Kirillov and R. Sakamoto, arXiv: 1406.1958v3 [math-ph].

[37] B. Lulek, T. Lulek, M. Labuz and R. Stagraczynski, Physica B 405 2654-2658 (2010).

[38] P. R. Giri, T. Deguchi, arXiv: 1411.5839 [hep-th]. 